the tumor, the subclavian artery is resected and reanastomosed end to end or with a ringed polytetrafluoroethylene (6- or 8-mm) graft.

The sternal component of the sternocleidomastoid muscle must be sutured back to the manubrium with heavy sutures when closing the incision. If the spinal accessory, dorsal scapular, and long thoracic nerves are preserved along with the trapezius, latissimus dorsi, rhomboid, levator scapulae, and serratus anterior muscles, this approach results in minimal shoulder dysfunction and cosmetic defect despite resection of the inner part of the clavicle (Figure 2).

\section{Discussion}

The anterior transclavicular approach with resection of the inner part of the clavicle and extension of the incision along the third rib gives excellent exposure from the thoracic inlet down to the pulmonary hilum. This approach allows dissection of the cervical structures and completion of the upper lobectomy and mediastinal lymph node dissection through the same incision under excellent conditions. Compared with the hemiclamshell approach, this approach provides excellent exposure to the distal subclavian vessels and allows direct anterior and anterolateral approach to the spine and brachial plexus. The anterior clavicular sparing approach described by several authors, including Grunenwald and Spag- giari, ${ }^{6}$ can provide adequate exposure to the thoracic inlet but offers limited view of the upper mediastinum and often requires an additional thoracotomy to perform the lobectomy and mediastinal lymph node dissection. If the inner part of the clavicle is removed, it is important, however, to preserve the spinal accessory, dorsal scapular, and long thoracic nerves in the posterior cervical triangle and to maintain the integrity of the trapezius, latissimus dorsi, rhomboid, and serratus anterior muscles by avoiding a posterolateral thoracotomy to obtain good cosmetic and functional results.

\section{References}

1. Dartevelle PG, Chapelier AR, Macchiarini P, Lenot B, Cerrina J, Le Roy Ladurie F, et al. Anterior transcervical-thoracic approach for radical resection of lung tumors invading the thoracic inlet. $J$ Thorac Cardiovasc Surg. 1993;105:1025-34.

2. Abbott LC, Lucas DB. The function of the clavicle. Ann Surg. 1954; 140:583-99.

3. Owens BD, Goss TP. The floating shoulder. J Bone Joint Surg. 2006; 88:1419-24.

4. Pouliart N, Gagey O. Significance of the latissimus dorsi for shoulder instability. Clin Anat. 2005;18:493-9.

5. Williams GR Jr, Shakil M, Klimkiewicz J, Iannotti JP. Anatomy of the scapulothoracic articulation. Clin Orthop Relat Res. 1999;359:237-46.

6. Grunenwald D, Spaggiari L. Transmanubrial osteomuscular sparing approach for apical chest tumors. Ann Thorac Surg. 1997;63:563-6.

\title{
Osteomalacia associated with a fibroblast growth factor-23 secreting chest wall tumor
}

\author{
Subroto Paul, MD, Michael Kurtz, BS, and Steven J. Mentzer, MD, Boston, Mass
}

\section{Clinical Summary}

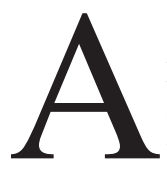
previously healthy 32-year-old man had a 9-month history of progressive rib, pelvic, and foot pain. Skeletal radiographs and bone scintigraphy revealed multiple fractures of the ribs, pelvis, and right foot.

Blood testing demonstrated low plasma phosphate levels (1.5 $\mathrm{mg} / \mathrm{dL}$; normal $2.4-5.0 \mathrm{mg} / \mathrm{dL})$ but normal calcium $(9.2 \mathrm{mg} / \mathrm{dL}$; normal $8.8-10.5 \mathrm{mg} / \mathrm{dL}$ ) and immunoreactive parathyroid hor-

From the Division of Thoracic Surgery, Brigham and Women's Hospital, Harvard Medical School, Boston, Mass.

Received for publication March 22, 2007; revisions received May 3, 2007; accepted for publication May 22, 2007.

Address for reprints: Steven J. Mentzer, MD, Room 259, Brigham \& Women's Hospital, 75 Francis S, Boston MA 02115 (E-mail: smentzer@ partners.org).

J Thorac Cardiovasc Surg 2007;134:803-5

$0022-5223 / \$ 32.00$

Copyright $\odot 2007$ by The American Association for Thoracic Surgery doi:10.1016/j.jtcvs.2007.05.025 mone levels (39 pg/mL; normal $11-80 \mathrm{pg} / \mathrm{mL}$ ). A 24-hour urine collection demonstrated phosphaturia (1593 mg/24 hours; normal 500-1200 mg/24 hours), suggesting a phosphate wasting disorder. Since 25 vitamin D (34 ng/mL; normal 20-57 ng/mL) and 1,25 vitamin $\mathrm{D}(19 \mathrm{pg} / \mathrm{mL} ; 18-62 \mathrm{pg} / \mathrm{mL})$ levels were within normal limits, a recently recognized disorder of paraneoplastic fibroblast growth factor (FGF)-23 production was investigated. Serum immunoassays for FGF-23 were initially found to be high normal (173 RU/mL; normal <180 RU/mL), but levels obtained the day of surgery were elevated (432 RU/mL).

As part of a systemic soft tissue and skeletal survey for an FGF-23 secreting tumor, a chest computed tomographic scan demonstrated an expansile and destructive lesion in the right posterior eighth rib (Figure 1, A). A fine needle aspiration biopsy revealed benign spindle cells with osteoclast-like cells and hemosiderin deposition.

Because no other bony or soft tissue tumors were identified, surgical resection of the chest wall tumor was recommended. At the time of surgery, thoracoscopic examination of the tumor demonstrated neovascularization of the parietal pleura (Figure 1, B). A complete excision of the chest wall mass required excision of a portion of the eighth rib (Figure 1,C). Gross evaluation of the tumor demonstrated multiple cystic spaces. The patient had an 

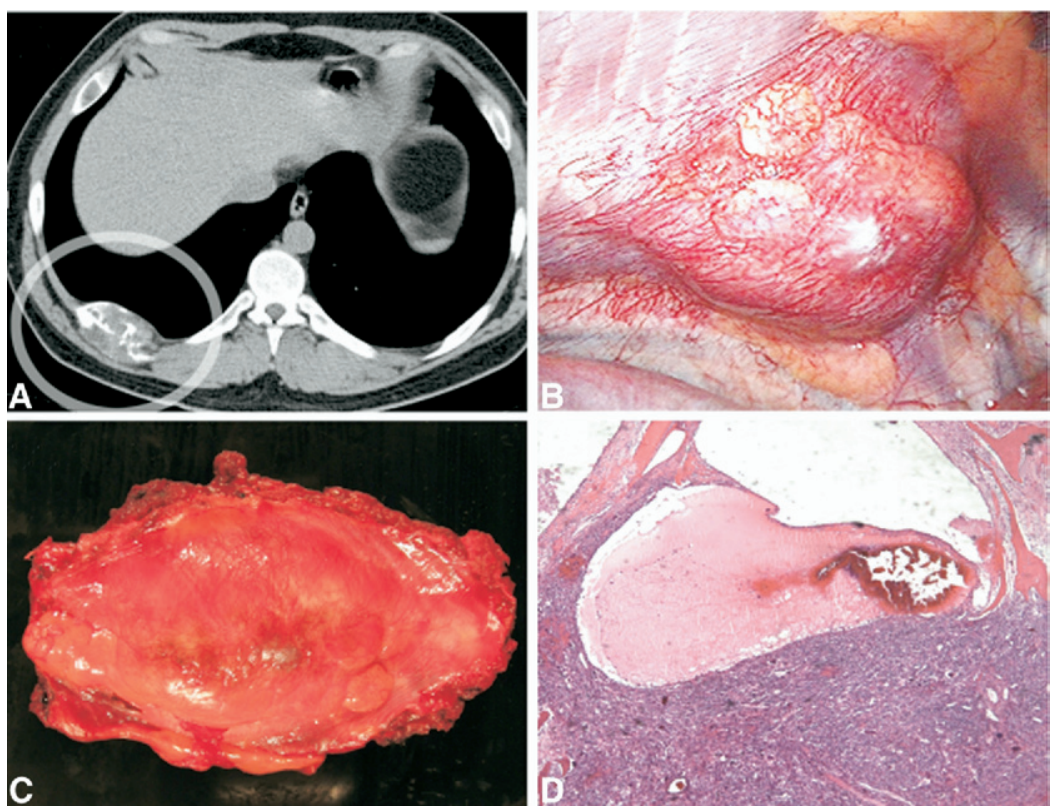

Figure 1. Patient with FGF-23 secreting tumor. A, Computed tomographic scan demonstrating a lesion in the right posterior chest wall (circle). B, Thoracoscopic appearance of the tumor: note the prominent neovascularization of the overlying parietal pleura. C, The lesion was completely excised with the limited resection of two ribs. D, Histopathologic analysis demonstrated spindle cells and aneurysmal bone cysts.

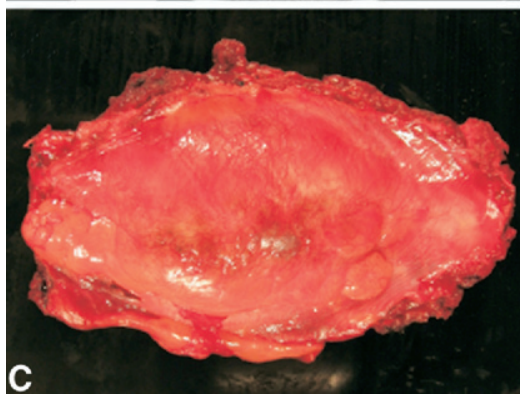

B.

uneventful recovery and was discharged on the second postoperative day.

Histopathologic examination of the mass revealed a spindle cell proliferation with features of an aneurysmal bone cyst (Figure 1, D). In the context of elevated FGF-23 levels, the histopathologic features were consistent with those of a phosphaturic mesenchymal tumor. Postoperatively, phosphate $(4.5 \mathrm{mg} / \mathrm{dL})$, urine phosphate $(1066 \mathrm{mg} / 24 \mathrm{~h}$ ), and FGF-23 (FGF-23 $<50 \mathrm{RU} / \mathrm{mL}$ to undetectable) levels normalized and remain normal 6 months postoperatively.

\section{Discussion}

Acquired "fragile bone syndromes" reflect abnormalities in calcium and phosphate metabolism. The primary regulators of calcium and phosphate homeostasis are vitamin D and parathyroid hormone. Vitamin D deficiency - whether a result of dietary insufficiency (ricketts), malabsorbtion, renal dysfunction or drug interactions-demonstrates a low level of the active form of vitamin $\mathrm{D}$ (1,25-dihydroxy vitamin $\mathrm{D}_{3}$; Calcitriol). In contrast, hyperparathyroidism will reflect an elevated immunoreactive parathyroid hormone level. In patients with normal vitamin D and parathyroid hormone serum levels, bone wasting may be due to a paraneoplastic etiology.

The constellation of an identifiable tumor and bone wasting has been referred to as oncogenic osteomalacia. Although the first report described a chest wall tumor, 104 of the 109 cases reviewed by Folpe and associates ${ }^{1}$ were found in the extremities, head, or neck. Isolated case reports have described tumors in the lung, mediastinum, thoracic spine, and rib. ${ }^{1}$ In a study of patients with oncogenic osteomalacia, Jonsson and colleagues ${ }^{2}$ have described 5 of 6 patients with markedly elevated levels of FGF-23.

With an improved understanding of their biologic activity, recent classification systems have described the tumors associated with oncogenic osteomalacia as phosphaturic mesenchymal tumor, mixed connective tissue variant or PMTMCT. ${ }^{3}$ Common to these tumors is a paraneoplastic syndrome associated with marked phosphaturia. PMTMCT tumors are locally expansile and destructive, but rarely associated with metastatic disease. When the primary tumor is not identifiable by conventional radiographic imaging, octreotide scanning may be useful to localize the site of the lesion. ${ }^{4}$

When an FGF-23 secreting tumor is identified, surgical resection is the treatment of choice. Preoperative therapy with octreotide has been advocated as a strategy to suppress the production of FGF-23 and minimize perioperative metabolic complications. Consistent with other reports, the margins of this tumor were readily assessed at surgery and a complete resection was obtained with limited morbidity.

The present case illustrates an improved understanding of both the etiology of oncogenic osteomalacia and the biology of tumorassociated angiogenesis. Recent studies have suggested that most cases of oncogenic osteomalacia are paraneoplastic syndromes associated with FGF-23 production. ${ }^{2}$ FGF-23 is a member of the FGF molecular family. FGF family members are involved in a variety of biologic processes including morphogenesis, tissue repair, and tumor growth and invasion. In this patient, the excessive production of FGF-23 may have contributed an autocrine effect; that is, FGF-23 may have contributed not only to pathologic posphaturia, but also to neovascularization and tumor progression. ${ }^{5}$ Regardless of the biologic effects, the paraneoplastic production of FGF-23 provides a useful serum marker for both diagnostic confirmation and postresectional surveillance.

\section{References}

1. Folpe AL, Fanburg-Smith JC, Billings SD, Bisceglia M, Bertoni F, Cho JY, et al. Most osteomalacia-associated mesenchymal tumors are a 
single histopathologic entity: an analysis of 32 cases and a comprehensive review of the literature. Am J Surg Pathol. 2004;28:1-30.

2. Jonsson KB, Zahradnik R, Larsson $\mathrm{T}$, White KE, Sugimoto T, Imanishi $\mathrm{Y}$, et al. Fibroblast growth factor 23 in oncogenic osteomalacia and X-linked hypophosphatemia. N Engl J Med. 2003;348: 1656-63.

3. Weidner N, Santa Cruz D. Phosphaturic mesenchymal tumors. A poly- morphous group causing osteomalacia or rickets. Cancer. 1987;59: 1442-54.

4. Seufert J, Ebert K, Muller J, Eulert J, Hendrich C, Werner E, et al. Octreotide therapy for tumor-induced osteomalacia. $N$ Engl $\mathrm{J}$ Med. 2001;345:1883-8.

5. Carpenter TO. Oncogenic osteomalacia-a complex dance of factors. N Engl J Med. 2003;348:1705-8.

\title{
Staged closure of tracheogastrocutaneous fistula after esophagectomy for infiltrative granular cell tumor
}

\author{
Robert Merritt, MD, ${ }^{a}$ Steven M. Zeitels, MD, ${ }^{a}$ William G. Austen, Jr, MD, ${ }^{a}$ Gregory Y. Lauwers, MD, ${ }^{b}$ and \\ Henning A. Gaissert, MD, ${ }^{a}$ Boston, Mass
}

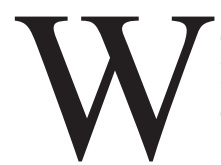

e report a tracheogastrocutaneous fistula and bilateral vocal cord paralysis after resection of an esophageal tumor. Reconstruction in 2 stages restored voice and swallowing.

\section{Clinical Summary}

A 22-year-old woman with a normal voice presented with a 3-year history of progressive dysphagia to solids and choking. A computed tomographic scan (Figure 1) showed a $3.8 \times 1.7-\mathrm{cm}$ esophageal wall mass compressing the trachea. A submucosal mass was found on endoscopy; biopsy was nondiagnostic. A cervical incisional biopsy specimen was interpreted as a benign granular cell tumor (GCT). The patient sought a second opinion and underwent neck re-exploration with tracheostomy. A second biopsy specimen was read as a malignant GCT. The mass was resected during a third procedure by dividing the trachea to provide access for total esophagectomy and posterior window resection of the trachea. After esophageal anastomosis in the neck to stomach, the cartilaginous trachea was closed and tracheal continuity was restored. The patient awoke hoarse from the operation. One week later, a fistula presented between the trachea and esophageal anastomosis.

In 4 additional procedures, skin flaps from the neck and left forearm were raised to close the fistula in the tracheal stoma, each of which was unsuccessful. The final attempt at closure 5 months after resection included division of the trachea with closure of the larynx and cutaneous end stoma. She presented to Massachusetts General Hospital requiring constant suction of gastric secretions from the tracheal stoma. Bilateral vocal cord palsy was noted on

From the Departments of Surgery ${ }^{\mathrm{a}}$ and Pathology, ${ }^{\mathrm{b}}$ Massachusetts General Hospital, Harvard Medical School, Boston, Mass.

Received for publication Feb 28, 2007; revisions received May 14, 2007; accepted for publication May 23, 2007.

Address for reprints: Henning A. Gaissert, MD, Massachusetts General Hospital, Blake 1570, Fruit St, Boston, MA 02114 (E-mail: hgaissert@partners.org).

J Thorac Cardiovasc Surg 2007;134:805-7

$0022-5223 / \$ 32.00$

Copyright $@ 2007$ by The American Association for Thoracic Surgery doi:10.1016/j.jtcvs.2007.05.029 laryngoscopy, and the subglottic lumen was closed below the cricoid. The 3-cm-long native esophagus ended in the 2-cm-wide fistula between the gastric mucosa and the membranous portion of the trachea. Gastric secretions freely washed into the trachea and drained from the cutaneous stoma. The residual trachea was $7 \mathrm{~cm}$ long, with inflamed mucosa.

The first stage of reconstruction closed the fistula 1 year after esophagectomy (Figure 2). By using a cervicomediastinal approach, the stomach was separated from the trachea, closed in the posterior mediastinum, and covered with pectoral muscle. Intestinal continuity was restored with substernal colon and colojejunostomy. Evaluation after recovery showed persistent vocal cord palsy in midabduction but no aspiration. The patient maintained her weight on an oral diet.

For tracheal reconstruction 5 months later, the cricoid was opened, and the distal trachea was mobilized for end-to-end anastomosis. After intubation for 3 days, a small tracheostomy was placed. The patient was discharged without stridor, leaving a stomal stent that was removed 4 months later. The patient now has a well-modulated voice without hoarseness. An uneventful lower body lift under endotracheal anesthesia has since been performed for cosmetic reasons.

Histologic review of the outside specimen at Massachusetts General Hospital established an infiltrative GCT of the esophagus (Figure 3). The tumor extensively involved the muscularis propria and adventitia and was present at the cervical and soft tissue

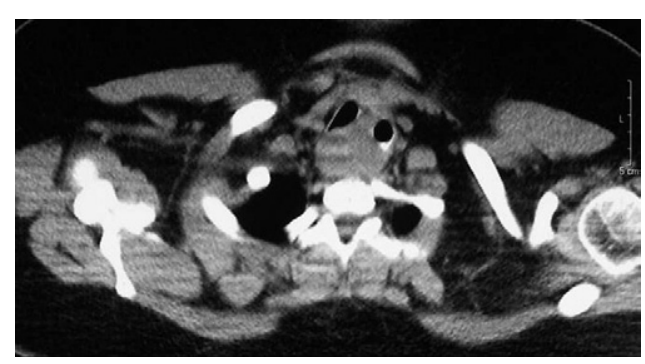

Figure 1. Computed tomographic scan demonstrates a cervical mass displacing both the tracheal and esophageal lumen. 